\title{
Synthesis, Characterization and Antibacterial Evaluation of some Novel Benzimidazole Derivatives Containing 1,3,4-thiadiazole moiety.
}

\section{MUAYED AHMED REDAYAN ${ }^{1 *}$, WASSAN BAQIR ALI ${ }^{2}$ and AHMED MUDHAFAR MOHAMMED ${ }^{2}$}

\author{
'Department of Chemistry, College of Education for Pure Sciences, Diyala University, Iraq. \\ ${ }^{2}$ Department of Chemistry, College of Sciences, Diyala University, Iraq. \\ ${ }^{*}$ Corresponding author E-mail: mredayan@gmail.com
}

http://dx.doi.org/10.13005/ojc/330656

(Received: August 04, 2017; Accepted: September 25, 2017)

\begin{abstract}
A series of novel 5-amino-1,3,4-thiadiazole-2-thiol and 1,3,4-thiadiazole-2,5-dithiol derivatives of benzimidazole were synthesized through nucleophilic substitution reaction of 5-substituted-2-(chloromethyl)-1H-benzimidazole, structures of the synthesized compounds were proved by spectral methods of analysis ( FT-IR, ${ }^{1} \mathrm{H}$ and ${ }^{13} \mathrm{C}$ NMR ). All the target compounds were screened for their antibacterial activity toward gram-negative (E.coli, P. aeruginosa) and Gram-positive (B. subtilis, $S$. aureus) bacteria, most of the synthesized derivatives exhibited good to moderate activity toward both Gram-positive (B. subtilis, S. aureus) and Gram-negative (E.coli, $P$. aeruginosa) bacteria.
\end{abstract}

Keywords: Benzimidazole, Bis benzimidazole, 1,3,4-thiadiazole, Antibacterial activity.

\section{INTRODUCTION}

The benzimidazole nucleus consider one of the most significant and important $\mathrm{N}$-containing fused organic compounds in a large number of synthetic pharmaceutical materials and natural products. ${ }^{1,2}$ Benzimidazole ring count a significant heterocyclic pharmacophore in the drug discovery, these compounds which carrying diverse substituents in the structure of benzimidazole are associated with a wide range of biological activities including: antifungal, ${ }^{3,4,5}$ anticancer, ${ }^{6,7,8}$ antiinflammatory, ${ }^{9,10}$ antioxidant, ${ }^{11,12,13}$ anti-bacterial, ${ }^{14,15,16,17}$ anti-viral, ${ }^{18,19}$ anticoagulant, ${ }^{20}$ and anti-hypertensive properties. ${ }^{21}$
Despite of many attempts to develop and discover new structural type in the search for great and more effective antimicrobials, benzimidazoles still consider as one of the most versatile and significant type of compounds against microbes.2,23

The benzimidazole containing compounds as a structural motif have been widely used in medicinal chemistry and drugs development. Amongst the benzimidazole derivatives 2-chloromethyl benzimidazole show a considerable importance in biological chemistry, they are important intermediates in the synthesis of many biologically active compounds. ${ }^{24}$ 
Designing new compounds in order to deal with resistant bacteria has become one of the most significant and great areas of antibacterial research today. Because the resistance of pathogenic bacteria toward common and available antimicrobial drugs is quickly becoming a major worldwide problem. So the discovery of new and potent antibacterial agent is more hallenging in current years. ${ }^{25}$

\section{MATERIALS AND METHODS}

Melting points were determined using stuart smp3 apparatus and are uncorrected, FT-IR spectra were recorded on shimadzu FT-IR spectrophotometer, ${ }^{1} \mathrm{H}$ and ${ }^{13} \mathrm{C}-\mathrm{NMR}$ spectra were recorded on brucker $300 \mathrm{MHz}$ spectrophotometer using (DMSO) as a solvent and TMS as internal reference. The compounds were checked for their purity on silica gel TLC plates and the visualization of spots performed by using UV light.

General method for the Synthesis of 5 substituted-2-(chloromethyl)-1H-benzimidazole $1(\mathrm{a}-\mathrm{d}) \mathbf{1 5}^{15,26,27}$

A mixture of 4-(un)substituted-o phenylenediamine (0.05 mole) and chloroacetic acid (0.05 mole) was dissolved in $(25 \mathrm{ml}) 4 \mathrm{~N} \mathrm{HCl}$ and refluxed for $4 \mathrm{hrs}$. The completion of the reaction was checked by using T.L.C (mobile phase:ethyl acetate: hexane $2: 1$ ). The reaction mixture was allowed to cool down and neutralized with ammonium hydroxide solution, the precipitate appeared was dried and recrystallized from (methanol/water).

\section{2-(chloromethyl)-1H-benzimidazole (1a):} yellow crystals, m.p: $147-150^{\circ} \mathrm{C}$, IR $(\mathrm{KBr}$, $\left.\mathrm{cm}^{-1}\right): \mathrm{N}-\mathrm{H}_{\text {str }}(3133)$, aromtic $\mathrm{C}-\mathrm{H}_{\text {str }}$ ( 3050), aliphatic $\mathrm{C}-\mathrm{H}_{\text {str }}(2900,2846), \mathrm{C}=\mathrm{N}_{\text {str }}$ (1625), aromatic $\mathrm{C}=\mathrm{C}_{\text {str }}$ $(1520,1446), \mathrm{C}^{-C l} \mathrm{str}_{\mathrm{str}}(640)$, Yield: $93 \%$.

\section{2-(chloromethyl)-5-methyl-1H-benzimidazole (1b):}

Dark brown crystals, m.p: $130-134^{\circ} \mathrm{C}$, IR $\left(\mathrm{KBr}, \mathrm{cm}^{-1}\right): \mathrm{N}-\mathrm{H}_{\text {str }}(3147)$, aromtic C-H $\mathrm{H}_{\text {str }}(3045)$, aliphatic $\mathrm{C}-\mathrm{H}_{\mathrm{str}}(2919,2850), \mathrm{C}=\mathrm{N}_{\mathrm{str}}(1622)$, aromatic $\mathrm{C}=\mathrm{C}_{\text {str }}(1522,1447), \mathrm{C}-\mathrm{Cl}_{\mathrm{str}}(646)$, yield: $90 \%$.

\section{2-(chloromethyl)-1 $\mathrm{H}$ - benzimidazole-5carboxylic} acid (1c):

Brown crystals, m.p: $290-293^{\circ} \mathrm{C}$, IR (KBr $\left.\mathrm{cm}^{-1}\right): \mathrm{N}-\mathrm{H}_{\text {str }}(3379), \mathrm{OH}_{\mathrm{str}}(2600-3350)$, aromtic
$\mathrm{C}-\mathrm{H}_{\text {str }}$ ( 3040), aliphatic $\mathrm{C}-\mathrm{H}_{\text {str }}(2966,2806), \mathrm{C}=\mathrm{O}_{\text {str }}$ (1681), $\mathrm{C}=\mathrm{N}_{\mathrm{str}}$ (1614), aromatic $\mathrm{C}=\mathrm{C}_{\mathrm{str}}(1425-1573)$, $\mathrm{C}-\mathrm{Cl}_{\mathrm{st}}(675)$, yield: $88 \%$.

2-(chloromethyl)-5-nitro-1 H-benzimidazole (1d): Dark yellow crystals, m.p:168-170 ${ }^{\circ} \mathrm{C}$, IR $\left(\mathrm{KBr}, \mathrm{cm}^{-1}\right): \mathrm{N}-\mathrm{H}_{\text {str }}(3259)$, aromtic $\mathrm{C}-\mathrm{H}_{\text {str }}$ (3028), aliphatic $\mathrm{C}-\mathrm{H}_{\mathrm{str}}(2980,2800), \mathrm{C}=\mathrm{N}_{\mathrm{str}}(1624), \mathrm{C}=\mathrm{C}_{\mathrm{str}}(1446$, 1471), $\mathrm{NO}_{2 s t}(1334,1508), \mathrm{C}_{\mathrm{str}} \mathrm{Cl}_{\mathrm{str}}(690)$, yield: $85 \%$.

\section{Synthesis of 5-amino-1,3,4-thiadiazole-2-thiol (2) ${ }^{28}$}

A mixture of (4 g, 0.04 mole) of thiosemicarbazide and $(4.66 \mathrm{~g}, 0.04$ mole) of anhydrous sodium carbonate were dissolved in 50 $\mathrm{ml}$ of absolute ethanol. ( $6.4 \mathrm{~g}, 0.08$ mole) of carbon disulfide was then added to this solution. The resulting mixture was then refluxed for $11 \mathrm{~h}$, subsequently the reaction mixture was allowed to cool down at room temperature. Most of solvent was distilled off under reduced pressure and the residue was dissolved in $40 \mathrm{~mL}$ of distilled water and then carefully acidified with cold conc. Hydrochloric acid to give pale yellow precipitate. The product was then filtered and washed with cold water, then recrystallized from hot water, T.L.C (mobile phase: ethyl acetate: hexane 2:1 ). m.p: 232$234^{\circ} \mathrm{C}$, IR $\left(\mathrm{KBr}, \mathrm{cm}^{-1}\right): \mathrm{N}-\mathrm{H}_{\text {str }}(3399,3279), \mathrm{S}-\mathrm{H}_{\text {str }}$ (2529), $\mathrm{C}=\mathrm{N}_{\mathrm{str}}$ (1601), $\mathrm{C}=\mathrm{S}_{\mathrm{str}}^{\text {str }}$ (1363), C-S $\mathrm{str}_{\text {str }}(670)$, yield $83 \%$.

\section{General method for the Synthesis of compounds} $3(a-d)^{29}$

5-amino-1,3,4-thiadiazole-2-thiol (2) (5 mmole) and fused sodium acetate ( $5 \mathrm{mmole}$ ) were added To the solution of compound (3) (5 mmole) in absolute ethanol $(35 \mathrm{ml})$, the mixture was refluxed for $12 \mathrm{~h}$, the reaction completion was checked by using T.L.C (mobile phase: ethyl acetate: hexane $2: 1$ ), then the reaction mixture was cooled and the precipitate was collected by filtration and dried.

5-[(1 H-benzimidazol-2-yl-methyl)sulfanyl]-1,3,4thiadiazol-2-amine (3a):

Yellow-brown crystals, m.p: $196-198^{\circ} \mathrm{C}$, IR $\left(\mathrm{KBr}, \mathrm{cm}^{-1}\right): \mathrm{NH}_{2}$ str $(3252)$, aromtic C-H $\mathrm{H}_{\text {str }}$ (3085), aliphatic $\mathrm{C}-\mathrm{H}_{\mathrm{str}}(2954,2918), \mathrm{C}=\mathrm{N}_{\mathrm{str}}(1622)$, aromatic $\mathrm{C}=\mathrm{C}_{\text {str }}(1526,1434), \mathrm{C}_{\mathrm{str}}(671)$, yield $53 \%$.

\section{5-\{[(5-methyl-1H-benzimidazol-2-yl) methyl]} sulfanyl\}-1,3,4-thiadiazol-2-amine (3b):

Brown crystals, m.p: $>350^{\circ} \mathrm{C}$, IR $(\mathrm{KBr}, \mathrm{cm}$ 1): $\mathrm{NH}_{2 \text { str }}(3342,3264), \mathrm{N}-\mathrm{H}_{\text {str }}(3133)$, aromtic $\mathrm{C}-\mathrm{H}_{\text {str }}$ 
(3080), aliphatic $C-\mathrm{H}_{\text {str }}(2972,2918), C=\mathrm{N}_{\text {str }}(1610)$, aromatic $\mathrm{C}=\mathrm{C}_{\mathrm{str}}(1556,1449), \mathrm{C}-\mathrm{S}_{\mathrm{str}}(621)$, yield $47 \%$.

2-\{[(5-amino-1,3,4-thiadiazol-2-yl) sulfanyl] methyl\}1H-benzimidazole-5-carboxylic acid (3c):

Brown crystals, m.p: 240 dec. ${ }^{\circ} \mathrm{C}$, IR (KBr, $\left.\mathrm{cm}^{-1}\right): \mathrm{OH}_{\mathrm{str}}(2800-3550), \mathrm{NH}_{2 \text { str }}(3442,3265), \mathrm{N}-\mathrm{H}_{\text {str }}$ (3147), aromtic $\mathrm{C}-\mathrm{H}_{\mathrm{str}}(3051)$, aliphatic $\mathrm{C}-\mathrm{H}_{\mathrm{str}}(2941$, 2864), $\mathrm{C}=\mathrm{O}_{\text {str }}$ (1697), $\mathrm{C}=\mathrm{N}_{\text {str }}(1616)$, aromatic $\mathrm{C}=\mathrm{C}_{\text {str }}$ (1571, 1512, 1421), C-S $\mathrm{strt}_{\text {str }}$ (671). ${ }^{1} \mathrm{HNMR}$ (DMSOd6) $\delta$ ppm: $3.9\left(\mathrm{~s}, 2 \mathrm{H}, \mathrm{CH}_{2}\right), 4.4(\mathrm{~s}, 1 \mathrm{H}, \mathrm{N}-\mathrm{H}), 4.58(\mathrm{~s}$, $\left.2 \mathrm{H}, \mathrm{NH}_{2}\right), 7.6-8.1(\mathrm{~m}, 3 \mathrm{H}, \mathrm{Ar}-\mathrm{H}), 12.9(\mathrm{~s}, 1 \mathrm{H}, \mathrm{OH})$, yield $45 \%$.

\section{5-\{[(5-nitro-1 H-benzimidazol-2-yl) methyl] sulfanyl\}-1,3,4-thiadiazol-2-amine (3d):}

Light brown crystals, m.p: $214^{\circ} \mathrm{C}$, IR $(\mathrm{KBr}$, $\left.\mathrm{cm}^{-1}\right): \mathrm{NH}_{2 \text { str }}(3406,3284), \mathrm{N}-\mathrm{H}_{\text {str }}(3107)$, aromtic C$\mathrm{H}_{\mathrm{str}}(3057)$, aliphatic $\mathrm{C}-\mathrm{H}_{\mathrm{str}}(2968,2800), \mathrm{C}=\mathrm{N}_{\mathrm{str}}$ (1627), aromatic $\mathrm{C}=\mathrm{C}_{\text {str }}(1537,1469), \mathrm{NO}_{2 \text { str }}$ (1338, 1514), C-S ${ }_{\text {str }}(640),{ }^{1} \mathrm{HNMR}$ (DMSO-d6) $\delta \mathrm{ppm}: 3.37$ $(\mathrm{s}, 1 \mathrm{H}, \mathrm{N}-\mathrm{H}), 4.3\left(\mathrm{~s}, 2 \mathrm{H}, \mathrm{CH}_{2}\right), 7\left(\mathrm{~s}, 2 \mathrm{H}, \mathrm{NH}_{2}\right), 7.6-$ $8.1(\mathrm{~m}, 3 \mathrm{H}, \mathrm{Ar}-\mathrm{H})$, yield $90 \%$.

\section{Synthesis of 1,3,4-thiadiazole-2,5-dithiol (4) ${ }^{30}$}

A mixture of carbon disulfide $(0.04$ mole, $30 \mathrm{~mL}$ ) and hydrazine hydrate $80 \%$ (0.04 mole, $10 \mathrm{~mL}$ ) with pyridine $(100 \mathrm{~mL})$ was heated under reflux for 5 hours. Then excess solvent was distilled off and the solid obtained was separated by adding (50 $\mathrm{mL}$ ) of water and $(10 \mathrm{~mL})$ of hydrochloric acid. The precipitate was then filtered, dried and recrystallized from ethanol. T.L.C (mobile phase: ethyl acetate: hexane 2:1). Yellow crystals, m.p: $163-166^{\circ} \mathrm{C}$, IR $\left(\mathrm{KBr}, \mathrm{cm}^{-1}\right): \mathrm{N}-\mathrm{H}_{\text {str }}(3055), \mathrm{S}-\mathrm{H}_{\text {str }}(2732), \mathrm{C}=\mathrm{N}_{\mathrm{str}}(1622)$, $\mathrm{C}=\mathrm{S}_{\mathrm{str}}(1266), \mathrm{C}-\mathrm{S}_{\mathrm{str}}(677)$, yield $85 \%$.

\section{General methods for the synthesis of compounds} $5(a-d)^{29}$

To the solution of compounds (1) (0.02mole ) in absolute ethanol $(60 \mathrm{ml})$ the $1,3,4-$ thiadiazole-2,5-dithiol (4) (0.01 mole) and fused sodium acetate $(0.02$ mole) were added and the mixture was refluxed for $12 \mathrm{hrs,} \mathrm{after} \mathrm{completion} \mathrm{of}$ the reaction which was checked by T.L.C (mobile phase: ethyl acetate: hexane 2:1) the mixture was allowed to cool down and cooled in ice bath, the product was collected by filtration and dried.

\section{2,2'-[1,3,4-thiadiazole-2,5-diylbis (sulfanediy} Imethanediyl)]bis(1 H-benzimidazole) (5a):

Yellow-brown crystals, m.p: $218-220$ dec. ${ }^{\circ} \mathrm{C}, \operatorname{IR}\left(\mathrm{KBr}, \mathrm{cm}^{-1}\right): \mathrm{N}-\mathrm{H}_{\mathrm{str}}(3420)$, aromtic $\mathrm{C}-\mathrm{H}_{\text {str }}$ (3100), aliphatic $\mathrm{C}-\mathrm{H}_{\mathrm{str}}(2978,2930), \mathrm{C}=\mathrm{N}_{\mathrm{str}}(1622)$, aromatic $\mathrm{C}=\mathrm{C}_{\mathrm{str}}(1526,1437), \mathrm{C}-\mathrm{S}_{\mathrm{str}}(746),{ }^{1} \mathrm{HNMR}$ (DMSO-d6) $\delta$ ppm: $3.4\left(\mathrm{~s}, 2 \mathrm{H}, \mathrm{CH}_{2}\right), 4.8(\mathrm{~s}, 1 \mathrm{H}, \mathrm{N}-\mathrm{H})$, 7.1-7.7 (m, 8H, Ar-H) yield $47 \%$.

2,2'-[1,3,4-thiadiazole-2,5-diylbis (sulfanediyImethanediyl)] bis(5-methyl-1 $H$-benzimidazole) (5b):

Light brown crystals, m.p: $>300^{\circ} \mathrm{C}, \mathrm{IR}(\mathrm{KBr}$, $\left.\mathrm{cm}^{-1}\right): \mathrm{N}-\mathrm{H}_{\mathrm{str}}(3417)$, aromtic $\mathrm{C}-\mathrm{H}_{\mathrm{str}}(3072)$, aliphatic $\mathrm{C}-\mathrm{H}_{\mathrm{str}}(2976), \mathrm{C}=\mathrm{N}_{\mathrm{str}}(1637)$, aromatic $\mathrm{C}=\mathrm{C}_{\mathrm{str}}(1618$, 1562), C-S str $(619)$, yield $44 \%$.

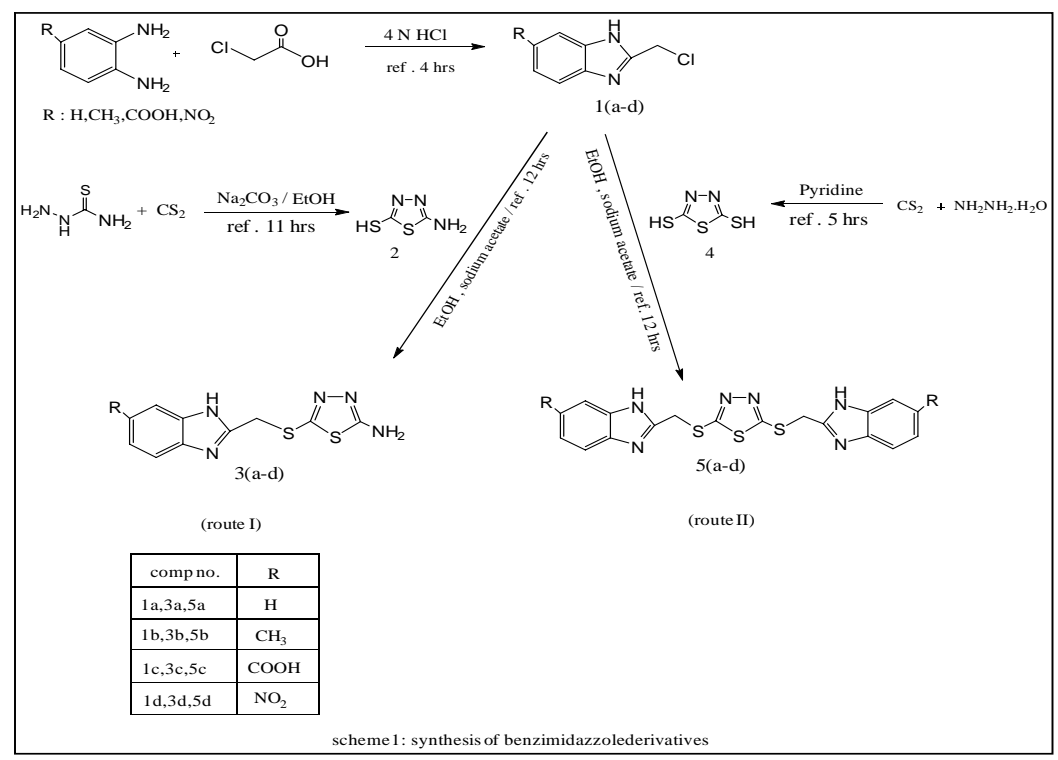


2,2'-[1,3,4-thiadiazole-2,5-diylbis (sulfanediylmethanediyl)]bis(1 H-benzimidazole-5-carboxylic acid) (5c):

Light green. M.p:284-285 ${ }^{\circ} \mathrm{C}, \mathrm{IR}\left(\mathrm{KBr}, \mathrm{cm}^{-1}\right)$ : N$\mathrm{H}_{\text {str }}(3431), \mathrm{OH}_{\text {str }}(2500-3600)$, aromtic C- $\mathrm{H}_{\text {str }}(3074)$, aliphatic $\mathrm{C}-\mathrm{H}_{\text {str }}(2974,2889,2804), \mathrm{C}=\mathrm{O}_{\text {str }}(1699)$, $\mathrm{C}=\mathrm{N}_{\mathrm{str}}(1631)$, aromatic $\mathrm{C}=\mathrm{C}_{\mathrm{str}}(1616,1564), \mathrm{C}-\mathrm{S}_{\mathrm{str}}$ (769), ${ }^{1} \mathrm{HNMR}$ (DMSO-d6) $\delta \mathrm{ppm}: 5.09$ (s, 2H, $\mathrm{CH}_{2}$ ), $5.3(\mathrm{~s}, 1 \mathrm{H}, \mathrm{N}-\mathrm{H})$, and 7.69-8.3 (m, 6H, Ar-H). ${ }^{13} \mathrm{CNMR}$ (DMSO-d6) $\delta$ ppm: $29.5\left(\mathrm{CH}_{2}\right), 114.3,116.2,124.5$, 126.7, 134.1, 137.1(Ar-C), 152.1(C=N of benzimidazole ), $164.3(\mathrm{C}=\mathrm{N}$ of thiadiazole , $167.2(\mathrm{C}=\mathrm{O})$, yield $73 \%$.

2,2'-[1,3,4-thiadiazole-2,5-diylbis (sulfanediyImethanediyl)]bis(5-nitro-1 H-benzimidazole)(5d): Light brown crystals, m.p: $165^{\circ} \mathrm{C}$. IR $(\mathrm{KBr}$, $\left.\mathrm{cm}^{-1}\right): \mathrm{N}-\mathrm{H}_{\text {str }}(3419)$, aromtic $\mathrm{C}-\mathrm{H}_{\mathrm{str}}$ (3099), aliphatic $\mathrm{C}-\mathrm{H}_{\text {str }}(2974,2918), \mathrm{C}=\mathrm{N}_{\text {str }}(1627)$, aromatic $\mathrm{C}=\mathrm{C}_{\text {str }}$ (1597, 1471$), \mathrm{NO}_{2 \text { str }}(1346,1519), \mathrm{C} \mathrm{S}_{\text {str }}(738)$, ${ }^{1} \mathrm{HNMR}$ (DMSO-d6) $\delta$ ppm: $\delta 3.6(\mathrm{~s}, 1 \mathrm{H}, \mathrm{N}-\mathrm{H})$, ä 5.0 (s, $\left.2 \mathrm{H}, \mathrm{CH}_{2}\right)$, and ä 7.6-8.4 ( $\left.\mathrm{m}, 6 \mathrm{H}, \mathrm{Ar}-\mathrm{H}\right) \cdot{ }^{13} \mathrm{CNMR}$ (DMSO-d6) $\delta$ ppm: $31.0\left(\mathrm{CH}_{2}\right), 104.7,108,116.1,117.9$, 118.2, 129.4 (Ar-C), 142.6(C=N of benzimidazole), 164.5 $(\mathrm{C}=\mathrm{N}$ of thiadiazole). Yield $78 \%$.

\section{RESULT AND DISCUSSION}

The reaction sequence for various title compounds is summerized in (scheme 1). The starting material 5-substituted-2-(chloromethyl)-1Hbenzimidazole 1(a-d) was synthesized according to a reported procedure through the reaction of 4-(un)substituted-o-phenylenediamine with chloroacetic acid. ${ }^{15,26,27}$ Structure of compounds 1 (ad) was confirmed by comparison of its physical and spectral data with the reported ones, ${ }^{26,27,31}$ nucleophilic substitution reaction of compound $+1(a-d)$ with 5-amino-1,3,4-thiadiazole-2-thiol (2) yielded 5-\{[(5substituted-1H-benzimidazol-2-yl)methyl]sulfanyl\}1,3,4-thiadiazol-2-amine 3(a-d) ( route I), the structure of compounds $3(\mathrm{a}-\mathrm{d})$ was confirmed by its IR, ${ }^{1} \mathrm{H}$ NMR, the IR spectra of the compound (3c) exhibited a broad band at (2800-3550 $\mathrm{cm}^{-1}$ for carboxylic $\mathrm{OH}$ ), (3442,3265 $\mathrm{cm}^{-1}$ for $\left.\mathrm{NH}_{2}\right)$, $\left(3147 \mathrm{~cm}^{-1}\right.$ for benzimidazole $\mathrm{N}-\mathrm{H}),\left(1697 \mathrm{~cm}^{-1}\right.$ for $\left.\mathrm{C}=\mathrm{O}\right)$ and band at $\left(671 \mathrm{~cm}^{-1}\right.$ for C-S ). ${ }^{1} \mathrm{HNMR}$ spectra showed the following chemical shifts at: $\delta 3.9\left(\mathrm{~s}, 2 \mathrm{H}, \mathrm{CH}_{2}\right), \delta 4.4(\mathrm{~s}, 1 \mathrm{H}, \mathrm{N}$ $\mathrm{H}), \delta 4.58\left(\mathrm{~s}, 2 \mathrm{H}, \mathrm{NH}_{2}\right), \delta 7.6 \delta 8.1(\mathrm{~m}, 3 \mathrm{H}, \mathrm{Ar}-\mathrm{H})$, and weak signal at $\delta 12.9$ for carboxylic $\mathrm{OH}$, and the IR spectra of the compound (3d) exhibited the following bands: $\left(3406,3284 \mathrm{~cm}^{-1}\right)$ for $\mathrm{NH}_{2},\left(3107 \mathrm{~cm}^{-1}\right)$ for benzimidazole $\mathrm{N}-\mathrm{H}$, and two peaks at $(1338,1323$ $\mathrm{cm}^{-1}$ ) corresponding for $\mathrm{NO}_{2}$ group, whereas the ${ }^{1} \mathrm{HNMR}$ spectra for this compound revealed the following peaks: $\delta 3.37(\mathrm{~s}, 1 \mathrm{H}, \mathrm{N}-\mathrm{H}), \delta 4.3(\mathrm{~s}, 2 \mathrm{H}$, $\left.\mathrm{CH}_{2}\right), \delta 7\left(\mathrm{~s}, 2 \mathrm{H}, \mathrm{NH}_{2}\right)$, and multiplet signals at $\delta$ (7.6-8.1) corresponding for aromatic protons.

Table. 1: Physical properties of the compounds

\begin{tabular}{|c|c|c|c|c|c|c|}
\hline $\begin{array}{l}\text { Compound } \\
\text { no. }\end{array}$ & $\mathrm{R}$ & m.p $\left({ }^{\circ} \mathrm{C}\right)$ & M.wt $(\mathrm{g} / \mathrm{mole})$ & Mol.Formula & Color & Yield \% \\
\hline$\overline{1 a}$ & $\mathrm{H}$ & $147-150$ & 166.61 & $\mathrm{C}_{8} \mathrm{H}_{7} \mathrm{CIN}_{2}$ & yellow & $93 \%$ \\
\hline $1 b$ & $\mathrm{CH}_{3}$ & $130-134$ & 180.64 & $\mathrm{C}_{9}^{8} \mathrm{H}_{9} \mathrm{CIN}_{2}^{2}$ & dark brown & $90 \%$ \\
\hline $1 c$ & $\mathrm{COOH}$ & $290-293$ & 210.62 & $\mathrm{C}_{9} \mathrm{H}_{7} \stackrel{\mathrm{CIN}}{2}_{2} \mathrm{O}_{2}$ & brown & $88 \%$ \\
\hline $1 d$ & $\mathrm{NO}_{2}$ & $168-170$ & 211.61 & $\mathrm{C}_{8} \mathrm{H}_{6} \mathrm{CIN}_{3} \mathrm{O}_{2}$ & dark yellow & $85 \%$ \\
\hline 2 & & $232-234$ & 133.19 & $\stackrel{\mathrm{C}}{2}_{2} \mathrm{H}_{3} \mathrm{~N}_{3}{ }_{3}$ & pale yellow & $83 \%$ \\
\hline $3 a$ & $\mathrm{H}$ & $196-198$ & 263.34 & $\mathrm{C}_{10} \mathrm{H}_{9}^{3} \mathrm{~N}_{5}^{3} \mathrm{~S}_{2}^{2}$ & yellow-brown & $53 \%$ \\
\hline $3 b$ & $\mathrm{CH}_{3}$ & $>350$ & 277.36 & $\mathrm{C}_{11} \mathrm{H}_{11} \mathrm{~N}_{5} \mathrm{~S}_{2}$ & brown & $47 \%$ \\
\hline $3 c$ & $\mathrm{COOH}$ & 240 dec. & 307.35 & $\mathrm{C}_{11} \mathrm{H}_{9} \mathrm{~N}_{5} \mathrm{~S}_{2}$ & brown & $45 \%$ \\
\hline $3 d$ & $\mathrm{NO}_{2}$ & 214 & 308.33 & $\mathrm{C}_{10} \mathrm{H}_{8} \mathrm{~N}_{6} \mathrm{O}_{2} \mathrm{~S}_{2}$ & light brown & $90 \%$ \\
\hline 4 & & $163-166$ & 150.23 & $\mathrm{C}_{2} \mathrm{H}_{2} \mathrm{~N}_{2} \mathrm{~S}_{3}$ & yellow & $85 \%$ \\
\hline $5 a$ & $\mathrm{H}$ & $218-220$ & 410.53 & $\mathrm{C}_{18} \mathrm{H}_{14}^{2} \mathrm{~N}_{6}^{2} \mathrm{~S}_{3}^{3}$ & yellow-brown & $47 \%$ \\
\hline $5 b$ & $\mathrm{CH}_{3}$ & $>300$ & 438.59 & $\mathrm{C}_{20} \mathrm{H}_{18}{ }_{18} \mathrm{~N}_{6} \mathrm{~S}_{3}$ & light brown & $44 \%$ \\
\hline $5 c$ & $\mathrm{COOH}$ & $284-285$ & 498.55 & $\mathrm{C}_{20} \mathrm{H}_{14} \mathrm{~N}_{6} \mathrm{O}_{4} \stackrel{\mathrm{S}}{3}_{3}$ & light green & $73 \%$ \\
\hline $5 d$ & $\mathrm{NO}_{2}$ & 165 & 500.53 & $\mathrm{C}_{18} \mathrm{H}_{12} \mathrm{~N}_{8} \mathrm{O}_{4} \mathrm{~S}_{3}$ & light brown & $78 \%$ \\
\hline
\end{tabular}

dec. : decomposed 
The ( route II ) also included nucleophilic substitution reaction of compound $1(\mathrm{a}-\mathrm{d})$ with $1,3,4$ thiadiazole-2,5-dithiol (4) yielded 2,2'-[1,3,4thiadiazole-2,5-diylbis (sulfanediyl methanediyl)] bis(5-subsituted-1H-benzimidazole) 5(a-d), The structure of compounds $5(\mathrm{a}-\mathrm{d})$ was confirmed by its IR, ${ }^{1} \mathrm{H}-\mathrm{NMR}$ and ${ }^{13} \mathrm{C}-\mathrm{NMR}$, The IR spectra of the compound (5c) showed a broad band at 2500-3600 $\mathrm{cm}^{-1}$ for carboxylic $\mathrm{OH}$ group, $3431 \mathrm{~cm}^{-1}$ for benzimidazole $\mathrm{N}-\mathrm{H}, 1699 \mathrm{~cm}^{-1}$ for $\mathrm{C}=\mathrm{O}$ group, $1631 \mathrm{~cm}^{-1}$ for $\mathrm{C}=\mathrm{N}$ group, and band at $769 \mathrm{~cm}^{-1}$ for C-S group. Whereas the ${ }^{1} \mathrm{HNMR}$ spectra exhibited the following chemical shifts: $\delta 5.09\left(\mathrm{~s}, 2 \mathrm{H}, \mathrm{CH}_{2}\right), \delta$ $5.3(\mathrm{~s}, 1 \mathrm{H}, \mathrm{N}-\mathrm{H})$, and $\delta 7.69-8.3(\mathrm{~m}, 6 \mathrm{H}, \mathrm{Ar}-\mathrm{H})$. The signals in ${ }^{13} \mathrm{CNMR}$ was appeared at around: $\delta 29.5$ accounted for the methylene group $\left(-\mathrm{CH}_{2}-\right)$, signals at $(114.3,116.2,124.5,126.7,134.1,137.1)$ could be for benzene ring, also ${ }^{13} \mathrm{CNMR}$ spectra showed signal about 152.1 and 164.3 for $(-C=N)$ of benzimidazole and thiadiazole ring respectively, whereas signal at 167.2 accounted for $(-\mathrm{C}=\mathrm{O})$ group.

The IR spectra for the compound (5d) found have the following bands: $3419 \mathrm{~cm}^{-1}$ for $\mathrm{N}-\mathrm{H}$ group , $1627 \mathrm{~cm}^{-1}$ for $\mathrm{C}=\mathrm{N}$ group, and two bands observed at $\left(1346,1315 \mathrm{~cm}^{-1}\right)$ accounted for $\mathrm{NO}_{2}$ group. The ${ }^{1}$ HNMR spectra showed the chemical shifts: $\delta 3.6(\mathrm{~s}, 1 \mathrm{H}, \mathrm{N}-\mathrm{H}), \delta 5.0\left(\mathrm{~s}, 2 \mathrm{H}, \mathrm{CH}_{2}\right)$, and peaks at $\delta 7.6-8.4(\mathrm{~m}, 6 \mathrm{H}, \mathrm{Ar}-\mathrm{H}) \cdot{ }^{13} \mathrm{CNMR}$ spectra result for this compound found in full agreement with its assigned structures. Beginning with signal appeared at about $\delta 31.0$ corresponding for the methylene group $\left(-\mathrm{CH}_{2}-\right)$, signals of benzene ring appeared at about $\delta(104.7,108,116.1,117.9,118.2$, 129.4) where as the chemical shifts at about 142.6 and 164.5 related to benzimidazole and thiadiazole $(-\mathrm{C}=\mathrm{N})$ group respectively. The purity of the synthesized compounds was monitored by TLC. Physical properties of the synthesized compounds are shown in Table 1.

\section{Antibacterial evaluation}

Some of the newly synthesized compounds were tested for their in-vitro antibacterial activities against Gram-negative including (Pseudomonas aeruginosa, Esherichia coli) and gram-positive including (Staphylococcus aureus, Bacillus subtilis) bacteria by disc diffusion method, the concentration of the compounds used were $(10 \mathrm{mg} / \mathrm{mL}$ and 100 $\mathrm{mg} / \mathrm{ml}$ ). Inhibition zones were measured in millimeters and compared with (Ampicillin and ciprofloxacin) as a standard antibiotics references. The results are illustrated in (Table 2 ) which

Table. 2 : Antibacterial activity of the synthesized compounds

\begin{tabular}{|c|c|c|c|c|c|}
\hline \multirow{3}{*}{$\begin{array}{l}\text { Compound } \\
\text { no. }\end{array}$} & \multirow{3}{*}{$\begin{array}{l}\text { Concentration } \\
(\mathrm{mg} / \mathrm{ml})\end{array}$} & \multicolumn{4}{|c|}{ Zone of inhibition ( in $\mathrm{mm}$ ) } \\
\hline & & \multicolumn{2}{|c|}{ Gram-positive } & \multicolumn{2}{|c|}{ Gram-negative } \\
\hline & & S. aureus & B. subtilis & $P$. aeruginosa & E. coli \\
\hline \multirow[t]{2}{*}{$3 a$} & 10 & 12 & 18 & 12 & 24 \\
\hline & 100 & 14 & 17 & - & 25 \\
\hline \multirow[t]{2}{*}{$3 b$} & 10 & - & - & - & - \\
\hline & 100 & 11 & 12 & - & - \\
\hline \multirow[t]{2}{*}{$3 c$} & 10 & - & - & 12 & 12 \\
\hline & 100 & 22 & 23 & 18 & 19 \\
\hline \multirow[t]{2}{*}{$3 d$} & 10 & 12 & 19 & 11 & 22 \\
\hline & 100 & 12 & 18 & - & 20 \\
\hline \multirow[t]{2}{*}{$5 a$} & 10 & - & 15 & - & 12 \\
\hline & 100 & - & - & - & - \\
\hline \multirow[t]{2}{*}{$5 b$} & 10 & - & 14 & - & - \\
\hline & 100 & 12 & 15 & - & - \\
\hline \multirow[t]{2}{*}{$5 c$} & 10 & 11 & 13 & 12 & 14 \\
\hline & 100 & 30 & 30 & 15 & 22 \\
\hline \multirow[t]{2}{*}{$5 d$} & 10 & - & 11 & - & 15 \\
\hline & 100 & - & - & - & - \\
\hline Ampicillin & & 22 & 23 & - & 10 \\
\hline ciprofloxacin & & 19 & 23 & 29 & - \\
\hline DMSO solvent & & 0 & 0 & 0 & 0 \\
\hline
\end{tabular}


demonstrates that most of compounds tested for their antibacterial activity exhibited good to moderate activities. Amongst all compounds tested (3a, 3c, 3d, 5c) showed good to moderate activity against all types of bacteria used.

\section{CONCLUSION}

Bnzimidazole derivatives containing 5-amino-1,3,4-thiadiazole-2-thiol and 1,3,4thiadiazole-2,5-dithiol were synthesized by nucleophilic substitution reaction of 5-substituted2-(chloromethyl)-1H-benzimidazole. The pharma- cological study was performed to determine the effects of substituent on the antibacterial activity, most of the derivatives showed good to moderate activity toward Gram-negative (E.coli, P.aeruginosa) and Gram-positive (B. subtilis, S. aureus) bacteria.

\section{ACKNOWLEDGMENT}

Authors are thankful to Chemistry department, college of education for pure sciences, Diyala University and also Chemistry department, Sciences college, Diyala University for their help and support.

\section{REFERENCES}

1. Bansal Y.; Silakari O., Bioorganic \& medicinal chemistry. 2012, 20, 6208-6236.

2. Saral H.; Özdamar Ö.; Uçar Ý., Journal of Molecular Structure. 2017, 1130,46-54.

3. Youssif B. G.; Abdel-Moty S. G.; Sayed I. M., Journal of Current Chemical and Pharmaceutical Sciences. 2014, 4,54-64.

4. Pawar N.; Dalal D.; Shimpi S.; Mahulikar P., European journal of pharmaceutical sciences 2004, 21,115-118.

5. Ansari K.; Lal C., European journal of medicinal chemistry. 2009, 44,4028-4033.

6. El-Nassan H. B., European journal of medicinal chemistry. 2012, 53,22-27.

7. Gellis A.; Kovacic H.; Boufatah N.; Vanelle P., European Journal of Medicinal Chemistry. 2008, 43,1858-1864.

8. Rashid M.; Husain A.; Mishra R., European journal of medicinal chemistry. 2012, 54,855-866.

9. Achar K. C.; Hosamani K. M.; Seetharamareddy H. R., European journal of medicinal chemistry. 2010, 45,2048-2054.

10. El-Nezhawy A. O.; Biuomy A. R.; Hassan F. S.; Ismaiel A. K.; Omar H. A., Bioorganic \& medicinal chemistry. 2013, 21, 1661-1670.

11. Ates-Alagoz Z.; Can-Eke B.; Coban T.; Iscan M.; Buyukbingol E., Archiv der Pharmazie. 2004, 337, 188-192.

12. Ayhan-KIlcigÝl G.; Kup C.; Çoban T.; CanEke B.; Özbey S.; Iscan M., Journal of enzyme inhibition and medicinal chemistry. 2005, 20,503-514.

13. Kálai T.; Balog M.; Szabó A.; Gulyás G.; Jeko J. z.; Sumegi B. z.; Hideg K., Journal of medicinal chemistry. 2009, 52, 1619-1629.

14. Nguyen P.; Baldeck J.; Olsson J.; Marquis R., Molecular Oral Microbiology. 2005, 20,93-100.

15. Gowda J.; Khader A.; Kalluraya B.; Hidayathulla S., Indian journal of chemistry. 2011, 50,1491-1495.

16. Kumar B.V.S.; Vaidya S.D.; Kumar R. V.; Bhirud S. B.; Mane R. B., European journal of medicinal chemistry. 2006, 41,599-604
17. Özkay Y.; Tunalý Y.; Karaca H.; Ipýkdað Ý., European journal of medicinal chemistry. 2010, 45,3293-3298.

18. Li Y.-F.; Wang G.-F.; He P.-L.; Huang W.-G.; Zhu F.-H.; Gao H.-Y.; Tang W.; Luo Y.; Feng C.L.; Shi L.-P., Journal of medicinal chemistry. 2006, 49, 4790-4794.

19. Vitale G.; Corona P.; Loriga M.; Carta A.; Paglietti G.; Giliberti G.; Sanna G.; Farci P.; Marongiu M. E.; La Colla P., European journal of medicinal chemistry. 2012, 53,83-97.

20. Mederski W. W.; Dorsch D.; Anzali S.; Gleitz J.; Cezanne B.; Tsaklakidis C., Bioorganic \& medicinal chemistry letters. 2004, 14,3763-3769.

21. Kumar J. R.; Jawahar L.; Pathak D., Journal of Chemistry. 2006, 3,278-285.

22. Ansari K.; Lal C., European journal of medicinal chemistry. 2009, 44,2294-2299.

23. Göker H.; Tunçbilek M.; Süzen S.; Kus C.; Altanlar N., Archiv der Pharmazie. 2001, 334,148-152.

24. El-Gohary N.; Shaaban M., European Journal of Medicinal Chemistry. 2017, 131,255-262.

25. Padalkar V. S.; Borse B. N.; Gupta V. D.; Phatangare K. R.; Patil V. S.; Umape P. G.; Sekar N., Arabian Journal of Chemistry. 2016, 9, S1125-S1130.

26. Mariappan G.; Hazarika R.; Alam F.; Karki R.; Patangia U.; Nath S., Arabian Journal of Chemistry. 2015, 8,715-719.

27. Galal S. A.; Hegab K. H.; Hashem A. M.; Youssef N. S., European journal of medicinal chemistry. 2010, 45,5685-5691.

28. Yusuf M.; Khan R. A.; Ahmed B., Bioorganic \& medicinal chemistry. 2008, 16,8029-8034.

29. Eisa H. M.; Barghash A.-e. M.; Badr S. M.; Farahat A. A., Indian journal of chemistry. 2010, 49,1515-1525.

30. Salimon J.; Salih N.; Hameed A.; Ibraheem H.; Yousif E., Journal of applied sciences research 2010, 6,866-870.

31. Petkar K.; Parekh P.; Kumari P. M. A.; baro A., Int. J. Pharm. Pharm. Sci. 2013, 5,115-119. 